\title{
New method for detecting surface defects of the rotational parts in remanufacturing service
}

\author{
Xiang Liu ${ }^{1, a}$, Xuhui Xia ${ }^{2, b}$, and Lei Wang ${ }^{2, c}$ \\ ${ }^{1}$ Engineering Training Centre, Wuhan University of Science and Technology, Wuhan, 430065, \\ China \\ ${ }^{2}$ School of Mechanical Engineering, Wuhan University of Science and Technology, Wuhan, 430081, \\ China \\ aemail:liuxiang@wust.edu.cn, bemail:xiaxuhui@wust.edu.cn, cemail:wanglei1987@wust.edu.cn
}

Keywords: remanufacturing service, surface defect, two-dimensional reciprocal cross entropy, image segmentation

\begin{abstract}
Remanufacturing is an important path to realize the sustainable development on modern industry. Defect detection is considered one of the most scientific and developed services in remanufacturing. Aiming to find surface defects of the rotational parts rapidly and accurately in the process of remanufacturing service, a new method based on improved two-dimensional reciprocal cross entropy is proposed in this paper. By using the adaptive morphological filtering, the 2D histogram is established, which enhances the anti-noise performance of the method. To accelarate the running time, the $2 \mathrm{D}$ reciprocal cross entropy threshold selection criterion function is decomposed into two independent 1D threshold selection criterion functions, then, the threshold determined by these two functions are jointed together to achieve the desired. Finally, through a case study of rollers, compared with one dimensional maximum entropy method, two-dimensional Otsu method and two-dimensional maximum entropy method, the practicability and effectiveness of the method is verified.
\end{abstract}

\section{Introduction}

Remanufacturing is an implementation of using high technology to repair and reconstruction the waste products, which uses scrap equipment in original spare parts with advanced industrial restoration and reconstruction method to make the remanufacturing product's quality and performance meet or exceed the new [1]. Rotating machinery parts are widely used in modern industry, however, they are rather liable to wear, cracks, holes or surface spalling after a long time working. Enterprises have to spend lots of money on replacing the damaged parts. By adopting remanufacturing, firms could make the cost down to the original $30 \% \sim 40 \%$ or so [2]. Due to the number of the recycled rotating parts in remanufacturing enterprises is huge, it is very important to detect surface defects accurately and rapidly. In the industrial sector, popular surface defect detection methods mainly include chemical penetration testing [3], infrared detection [4] [5], eddy current testing [6] [7] and so on. However, all these methods mentioned above have their limitations in the filed of remanufacturing. The development of machine vision based on computer technology provides new ways to solve this problem [8] [9] [10].

A complete discovery and segmentation of defects is the key to surface detection method, whose purpose is to find an appropriate threshold to make the image get the best segmentation results. The method proposed by Otsu [11] is one of the most classical algorithms in this area, which has been used to date. Kapur et al. [12] put forward a new way that using one-dimensional maximum entropy for image segmentation. Due to the good segmentation effect and simple realization, it has become a commonly used way to select the threshold of the segmentation. However, these methods' effect is not ideal, in the case of noise. Abutaleb [13] proposed a two-dimensional entropy threshold method, experiments show that 2D entropy threshold method has stronger anti-noise performance, but the computational complexity significantly increased. In order to reduce the computation of 2D entropy threshold, many fast algorithms have been proposed. In general, these fast algorithms can 
be divided into two major categories. One method considers searching threshold as a typical optimization problem [14] [15]. A variety of intelligent optimization algorithms are usually adopted to solve the problem. While others [16] [17] improve the computational speed by using iterative recursion method. What got more attention, however, was that Li [18] subsequently introduced the concept of cross entropy into the filed of image segmentation, and presented a threshold segmentation algorithm based on minimum cross entropy. His work showed that the segmentation effect of cross entropy is better than that of Otsu's method. Xue et al. [19] considered the mean difference of the posterior probability between the target and the background, and then proposed a maximum cross entropy algorithm based on the maximum difference. As the segmentation algorithm based on cross entropy has good validity, rationality and robustness, it has attracted widespread attention. Recently, Wu et al. defined a reciprocal cross entropy in the literature [20], and applied it in multi-threshold segmentation of flame image. As the reciprocal cross entropy abandons the logarithm operation, it is more liable to apply it in the occasion of higher real-time requirement.

Although the reciprocal cross entropy has the advantage in calculating speed, it has some defects in its definition. Aiming at the defects of reciprocal cross entropy, an improved method which can avoid the calculation of no insignificant value is proposed in this paper. Taking into account the uncertainty of image noise, a new way to establish two-dimensional histogram based on adaptive morphological filtering is applied. When calculating the threshold vector of segmentation criterion function, the two dimensional criterion function is decomposed into two independent one dimensional threshold criterion function, which makes the complexity of the computation decreased from $O\left(L^{4}\right)$ to $O(L)$. Finally, the effectiveness and superiority of the method are verified by a case study of roller's surface detection.

\section{Improved reciprocal cross entropy}

Assuming that $P$ and $Q$ are two distribution functions denoted as: $P=\left\{p_{i}\right\}, Q=\left\{q_{i}\right\}$, where $p_{i} \geq 0$, $q_{i} \geq 0, \quad i=1,2, \cdots, N$ and $\sum_{i=1}^{N} p_{i}=1, \sum_{i=1}^{N} q_{i}=1$. Then, the distance between the two distributions can be measured as:

$$
D(P, Q)=\sum_{i=1}^{N} q_{i} \log _{2} \frac{q_{i}}{p_{i}}
$$

According to (1), literature [20] proposed a definition of reciprocal cross entropy as follows:

$$
D(P, Q)=1-2 \sum_{i=1}^{N} p_{i} \frac{1}{1+\frac{p_{i}}{q_{i}}}
$$

It's not difficult to find that (2) is no sense when $q_{\mathrm{i}}=0$. So, an improved reciprocal cross entropy is proposed in the form of (3).

$$
D(P, Q)=1-\frac{2}{1+N \alpha} \sum_{i=1}^{N}\left(p_{i}+\alpha\right) \frac{1}{(1+N \alpha)+\left(\frac{p_{i}+\alpha}{q_{i}+\alpha}\right)}
$$

Where $\alpha$ is a constant between 0 and 1 .

Since the information entropy can be understood as the occurrence probability of some specific information, the value range of the entropy function should be between 0 and 1 . That means the value of (3) must between 0 and 1 . The proof as follows:

Proof

$$
\text { since } \sum_{i=1}^{N} p_{i}=1, \sum_{i=1}^{N} q_{i}=1 \quad \therefore \sum_{i=1}^{N} p_{i}^{\prime}=\sum_{i=1}^{N} q_{i}^{\prime}=1+N \alpha
$$




$$
\begin{aligned}
& \text { let } D^{\prime}(P, Q)=(1+N \alpha)-2 \sum_{i=1}^{N}\left(p_{i}+\alpha\right) \frac{1}{(1+N \alpha)+\left(\frac{p_{i+\alpha}}{q_{i}+\alpha}\right)}=(1+N \alpha)-\sum_{i=1}^{N}\left(q_{i}+\alpha\right) \frac{2\left(\frac{p_{i}+\alpha}{q_{i}+\alpha}\right)}{(1+N \alpha)+\left(\frac{p_{i}+\alpha}{q_{i}+\alpha}\right)} \\
& \therefore \quad D^{\prime}(P, Q)=(1+N \alpha)-\sum_{i=1}^{N} \frac{2\left(\frac{p_{i}^{\prime}}{q_{i}^{\prime}}\right)}{(1+N \alpha)+\left(\frac{p_{i}^{\prime}}{q_{i}^{\prime}}\right)} \\
& \text { let } F(x)=1-\frac{2 x}{(1+N \alpha)+x} \text {, where } x_{i}=\frac{p_{i}^{\prime}}{q_{i}^{\prime}} \\
& \text { then } D^{\prime}(P, Q)=\sum_{i=1}^{N} q_{i}^{\prime} F\left(x_{i}\right)
\end{aligned}
$$

According to Jensen's inequality, we have

$$
\begin{aligned}
& D^{\prime}(P, Q)=\sum_{i=1}^{N} q_{i}^{\prime} F\left(x_{i}\right) \geq \sum_{i=1}^{N} F\left(q_{i}^{\prime} x_{i}\right)=1-\frac{2 \sum_{i=1}^{N} p_{i}^{\prime}}{(1+N \alpha)+\sum_{i=1}^{N} p_{i}^{\prime}}=1-\frac{2(1+N \alpha)}{(1+N \alpha)+(1+N \alpha)}=1-1=0 \\
& \text { let } G(x)=\frac{2 x}{(1+N \alpha)+x} \text {, where } x>0
\end{aligned}
$$

Obviously, $\mathrm{G}(\mathrm{x})$ is a monotonically increasing function in the domain of definition, and $\mathrm{G}(\mathrm{x})>0$.

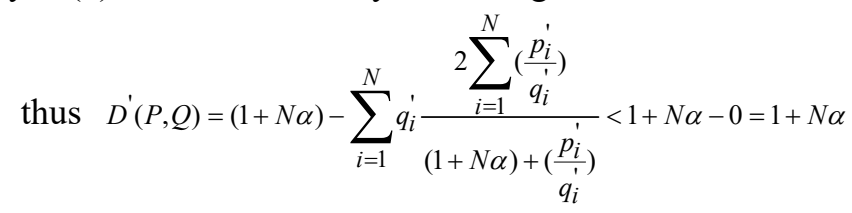

$0 \leq D^{\prime}(P, Q)<1+N \alpha$

considering that $D(P, Q)=\frac{1}{1+N \alpha} D^{\prime}(P, Q)$

Then, $D(P, Q) \in[0,1)$

By comparing (2) with (3), it can be concluded that (2) is a special case of (3) under condition that $\alpha=0$. In other word, (3) is a universal form of reciprocal cross entropy.

\section{Criterion function of image segmentation}

The following discussion have been divided into both cases: one-dimensional scale and two-dimensional scale.

\section{One-dimensional criterion function}

The following notations are adopted in this section:

$M \quad$ Width of a image

$N \quad$ Height of a image

$f(m, n) \quad$ Gray value of the pixel $(\mathrm{m}, \mathrm{n})$

$L \quad$ Gray levels of a image

$H(i) \quad$ Histogram function of a image

$p(i) \quad$ The probability of the pixel with a gray level of $i$

$t \quad$ Value of the threshold

$C_{o} \quad$ Object class in a image

$C_{b} \quad$ Background class in a image

$\omega_{o} \quad$ Prior probability of the object class

$\omega_{b} \quad$ Prior probability of the background class

$\mu_{o} \quad$ Mean value of the object class

$\mu_{b} \quad$ Mean value of the background class

$\mu_{T} \quad$ Mean value of a image

$C_{T} \quad$ A set of all the pixels in a image 
$S \quad$ Sum of all the pixels in a image

Assuming that a threshold $t$ divides a image into two classes, in which the gray value of the object class is less than that of background class, then the two classes can be expressed as:

$$
\begin{aligned}
& C_{O}=\{f(m, n) \mid f(m, n)=0,1, \cdots, t ; m \in[1, M], n \in[1, N]\} \\
& C_{b}=\{f(m, n) \mid f(m, n)=t+1, t+2, \cdots, L-1 ; m \in[1, M], n \in[1, N]\}
\end{aligned}
$$

Following the definition above, it is not difficult to derive these expressions below:

$$
\begin{aligned}
& p(i)=H(i) / M \times N, \text { let } T=M \times N \text {, then } p(i)=H(i) / T \text {, where } \sum_{i=0}^{L-1} p(i)=1 \\
& \omega_{o}(t)=\sum_{i=0}^{t} p(i)=\frac{1}{T} \sum_{i=0}^{t} H(i), \omega_{b}(t)=\sum_{i=t+1}^{L-1} p(i)=\frac{1}{T} \sum_{i=t+1}^{L-1} H(i) \\
& \mu_{o}(t)=\frac{\sum_{i=0}^{t} i p(i)}{\sum_{i=0}^{t} p(i)}=\frac{\sum_{i=0}^{t} i p(i)}{\omega_{o}(t)}, \mu_{b}(t)=\frac{\sum_{i=t+1}^{L-1} i p(i)}{\sum_{i=t+1}^{L-1} p(i)}=\frac{\sum_{i=t+1}}{\omega_{b}(t)} \\
& \mu_{T}=\mu_{O}(t)+\mu_{b}(t)=\frac{1}{T} \sum_{f(x, y) \in C_{T}} f(x, y)=\omega_{o}(t) \mu_{o}(t)+\omega_{b}(t) \mu_{b}(t) \\
& S=\sum_{f(x, y) \in C_{T}} f(x, y)=\mu_{T} \cdot T
\end{aligned}
$$

let $\quad p_{m, n}=\frac{f(m, n)}{\sum_{f(x, y) \in C_{T}} f(x, y)}=\frac{f(m, n)}{S}, \quad q_{m, n}= \begin{cases}\frac{\mu_{o}(t)}{\sum_{f(x, y) \in C_{T}} f(x, y)}=\frac{\mu_{o}(t)}{S}, & f(m, n) \in C_{o} \\ \frac{\mu_{b}(t)}{\sum_{f(x, y) \in C_{T}} f(x, y)}=\frac{\mu_{b}(t)}{S}, & f(m, n) \in C_{b} .\end{cases}$

It is obviously that both $p_{\mathrm{m}, \mathrm{n}}$ and $q_{\mathrm{m}, \mathrm{n}}$ are non-negative. They also meet the following conditions:

$$
\begin{aligned}
& \sum_{f(x, y) \in C_{T}} p_{m, n}=1 \\
& \sum_{f(x, y) \in C_{T}, n} q_{m}=\frac{1}{S}\left[\left(\sum_{i=0}^{t} H(i)\right) \mu_{o}(t)+\left(\sum_{i=t+1}^{L-1} H(i)\right) \mu_{b}(t)\right]=1
\end{aligned}
$$

This indicates that $p_{m, n}$ and $q_{m, n}$ are in accord with the conditions of (3). Therefore, the reciprocal cross entropy between the two distributions can be expressed as:

$$
\begin{aligned}
D(P, Q)=1-\frac{2}{(1+L \alpha) S T} & \left(\sum_{i=0}^{t} p(i)(i+S \alpha) \frac{1}{(1+L \alpha)+\left(\frac{i+S \alpha}{\mu_{o}(t)+S \alpha}\right)}\right. \\
& \left.+\sum_{i=t+1}^{L-1} p(i)(i+S \alpha) \frac{1}{(1+L \alpha)+\left(\frac{i+S \alpha}{\mu_{b}(t)+S \alpha}\right)}\right)
\end{aligned}
$$

The difference of the information between the two distributions is minimum when the cross entropy of the image is minimum. Follow this principle, selecting the value of the parameter $t$ which minimize the cross entropy as the threshold of image segmentation. Considering that the value of $2 /(1+L \alpha) S T$ is a constant, to minimize (4) is equivalent to maximize (5) below. 


$$
\begin{aligned}
\eta(t)= & \sum_{i=0}^{t} p(i)(i+S \alpha) \frac{1}{(1+L \alpha)+\left(\frac{i+S \alpha}{\mu_{o}(t)+S \alpha}\right)} \\
& +\sum_{i=t+1}^{L-1} p(i)(i+S \alpha) \frac{1}{(1+L \alpha)+\left(\frac{i+S \alpha}{\mu_{b}(t)+S \alpha}\right)}
\end{aligned}
$$

The optimal threshold of one-dimensional reciprocal cross entropy threshold segmentation should meet the following expression:

$$
t^{*}=\underset{t \in[0, L-1]}{\operatorname{Arg} \max }\{\eta(t)\}
$$

\section{Two-dimensional criterion function}

Here is a list of notations which are adopted in the discussion of this section:

$\begin{array}{ll}M & \text { Width of a image } \\ N & \text { Height of a image } \\ L & \text { Gray levels of a image } \\ p(i, j) & \text { Joint probability distribution of a pixel pair }(i, j) \\ H(i, j) & \text { Two-dimensional histogram function } \\ (t, s) & \quad \text { Threshold vector } \\ \omega_{\mathrm{o}} & \text { Prior probability of the object class } \\ \omega_{\mathrm{b}} & \text { Prior probability of the background class } \\ \mu_{\mathbf{0}} & \text { Mean vector of the object class } \\ \mu_{\mathrm{b}} & \text { Mean vector of the background class }\end{array}$

Different from the methods in most literatures, this paper uses a method of adaptive morphological filter with multi-scale and multi-structure elements to establish 2D histogram(H(i, j)). As a matter of fact, most literatures use a gray pixel and its 3-by-3 neighborhood average (or neighborhood median) to establish 2D histogram. The essence is to enhance the anti-noise ability of the segmentation algorithm by average filtering or median filtering. But both mean filtering and median filtering have their limitations in denoising [21], which makes the image segmentation getting worse. The following case study shows the proposed method can greatly improve the anti-noise performance. The specific steps of adaptive morphological filtering are as follows:

Step 1: Choosing structure elements that different in scales but same in directions for morphological "open-close" filtering. Noting that, this filtering processing should in accordance with the order from low-scale to high-sacle. The structure elements that adopted in this thesis are shown in Fig. 1.

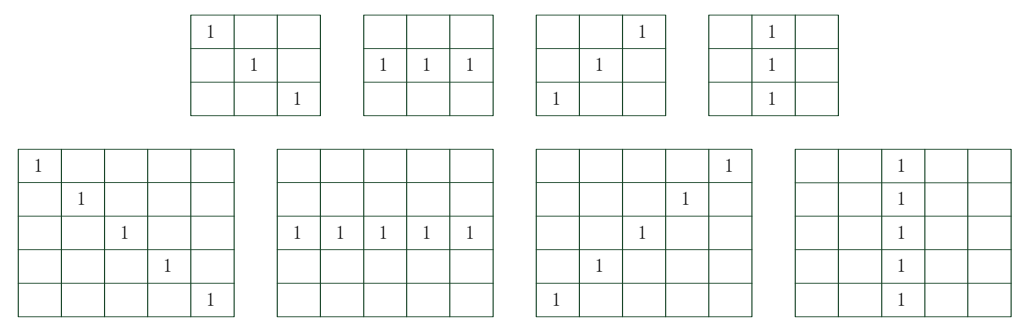

Fig. 1 Schematic diagram of the structure elements of the two scales

Step 2: Determining the weights of the filtered results in each direction. Let $n_{1}, n_{2}, n_{3}$ and $n_{4}$ respectively represent the number that the structure elements in each direction could "be filled" in the image. Then, the normalized weight $\alpha_{1}, \alpha_{2}, \alpha_{3}$ and $\alpha_{4}$ can be defined as (7).

$$
\left\{\begin{array}{l}
\alpha_{1}=n_{1} /\left(n_{1}+n_{2}+n_{3}+n_{4}\right) \\
\alpha_{2}=n_{2} /\left(n_{1}+n_{2}+n_{3}+n_{4}\right) \\
\alpha_{3}=n_{3} /\left(n_{1}+n_{2}+n_{3}+n_{4}\right) \\
\alpha_{4}=n_{4} /\left(n_{1}+n_{2}+n_{3}+n_{4}\right)
\end{array}\right.
$$

Step 3: Weighting the results of each direction according to their respective weights. Let fi represent the results of each direction, where $\mathrm{i}=1,2,3,4$. Then, the final result is obtained by (8). 
These three steps above can be clearly illustrated by Fig. 2 .

$$
F=\sum_{i=1}^{4} \alpha_{i} f_{i}
$$

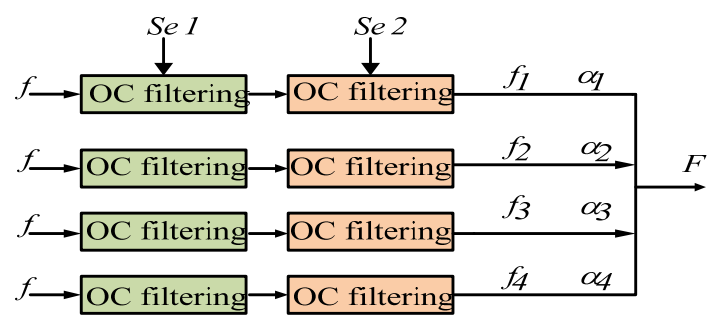

Fig. 2 Schematic diagram of adaptive morphological filtering with multi-scale and multi-structure elements

Using $H(i, j)$ to represent the 2D histogram function, it can be interpreted as the number of pixel pair which has a gray value of $i$ in orginal image, while that of $j$ in morphological filtered image. Similar to the one dimensional case, it is easy to obtain the joint probability distribution expression: $p(i, j)=H(i, j) / M \times N$, where $p(i, j) \geq 0$ and $\sum_{i=0}^{L-1} \sum_{j=0}^{L-1} p(i, j)=1$.

In Fig. 3(a), the horizontal axis represents the gray level of original image, while the vertical axis represents the gray level of morphologically processed image. A threshold vector then divides the 2D histogram into 4 regions, which have already been marked different labels in Fig. 3(a). Pixels in continuous areas of a image will be distributed along the main diagonal in the 2D histogram, as shown in Fig. 3(b). So it can be inferred that Region 1 \& 3 represent the object class, while Region $2 \& 4$ represent the background class.

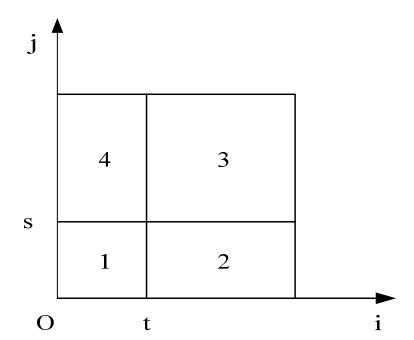

(a)

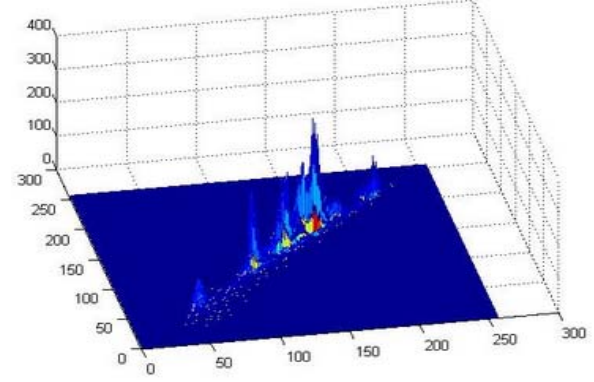

(b)

Fig. 3 Sketch map of two-dimensional histogram and its segmentation region

Similar to the derivation method of (5), we get the 2D reciprocal cross entropy criterion function, as shown in (9).

$$
\begin{aligned}
& \eta(t, s)=\sum_{i=0}^{t} \sum_{j=0}^{s} p(i, j)\left[\frac{(i+S \alpha)}{(1+L \alpha)+\frac{i+S \alpha}{\mu_{o i}(t, s)+S \alpha}}+\frac{(j+S \alpha)}{(1+L \alpha)+\frac{j+S \alpha}{\mu_{o j}(t, s)+S \alpha}}\right] \\
& +\sum_{i=t+1}^{L-1} \sum_{j=s+1}^{L-1} p(i, j)\left[\frac{(i+S \alpha)}{(1+L \alpha)+\frac{i+S \alpha}{\mu_{b i}(t, s)+S \alpha}}+\frac{(j+S \alpha)}{(1+L \alpha)+\frac{j+S \alpha}{\mu_{b j}(t, s)+S \alpha}}\right]
\end{aligned}
$$

Where, $\boldsymbol{\mu}_{\boldsymbol{o}}=\left[\mu_{o i}, \mu_{o j}\right]^{T}, \boldsymbol{\mu}_{\boldsymbol{b}}=\left[\mu_{b i}, \mu_{b j}\right]^{T}$

$$
\begin{aligned}
& \mu_{o i}(t, s)=\sum_{i=0}^{t} \sum_{j=0}^{s} i p(i, j) / \omega_{o}(t, s), \quad \mu_{o j}(t, s)=\sum_{i=0}^{t} \sum_{j=0}^{s} j p(i, j) / \omega_{o}(t, s) \\
& \mu_{b i}(t, s)=\sum_{i=t+1}^{L-1} \sum_{j=s+1}^{L-1} i p(i, j) / \omega_{b}(t, s), \mu_{b j}(t, s)=\sum_{i=t+1}^{L-1} \sum_{j=s+1}^{L-1} j p(i, j) / \omega_{b}(t, s)
\end{aligned}
$$




$$
\omega_{o}(t, s)=\sum_{i=0}^{t} \sum_{j=0}^{s} p(i, j), \quad \omega_{b}(t, s)=\sum_{i=t+1}^{L-1} \sum_{j=s+1}^{L-1} p(i, j)
$$

The optimal threshold vector $\left(t^{*}, s^{*}\right)$ for 2D reciprocal cross entropy threshold segmentation should be satisfied with:

$$
\left(t^{*}, s^{*}\right)=\operatorname{Arg} \max _{t, s \in[0, L-1]}\{\eta(t, s)\}
$$

\section{Method for solving the optimal threshold vector}

Through the analysis of (9), the computational complexity will be reached $O\left(L^{4}\right)$, if the exhaustive method is adopted to calculate the optimal threshold vector. At this point, it has trouble to implement real-time image processing Yue et al. [22] put forward a method of reducing dimension when solving the optimal threshold of two-dimensional Otsu method. This method decrease the computational complexity to $O(L)$ by combining two one-dimensional threshold to form a two-dimensional threshold vector. Yue also proved that the threshold vector obtained by reducing dimension method is the same as that obtained by two-dimensional Otsu method, which is based on the assumption that ignore the noise and edge regions in the $2 \mathrm{D}$ histogram away from the main diagonal. Now assuming that in Fig. 3(a), the probability of the pixel distribution in Region 2 $\& 4$ are zero. Then, we have:

$$
\begin{aligned}
& \mu_{o i}(t, s)=\sum_{i=0}^{t} \sum_{j=0}^{s} i p(i, j) / \omega_{o}(t, s)=\sum_{i=0}^{t} \sum_{j=0}^{L-1} i p(i, j) / \sum_{i=0}^{t} \sum_{j=0}^{L-1} p(i, j)=\sum_{i=0}^{t} i V_{i} / \sum_{i=0}^{t} V_{i}=\mu_{o}(t) \\
& \mu_{o j}(t, s)=\sum_{i=0}^{t} \sum_{j=0}^{s} j p(i, j) / \omega_{o}(t, s)=\sum_{i=0}^{L-1} \sum_{j=0}^{s} j p(i, j) / \sum_{i=0}^{L-1} \sum_{j=0}^{s} p(i, j)=\sum_{j=0}^{s} j H_{j} / \sum_{j=0}^{s} H_{j}=\mu_{o}(s) \\
& \text { Where, } V_{i}=\sum_{j=0}^{L-1} p(i, j), H_{j}=\sum_{i=0}^{L-1} p(i, j) .
\end{aligned}
$$

It is evident that $V_{i}$ is a marginal probability distribution of joint probability distribution with respect to the parameter $i$, while $H_{j}$ is a marginal probability distribution of joint probability distribution with respect to the parameter $j$. In the same way, it is not difficult to achieve the following results: $\mu_{b i}(t, s)=\mu_{b}(t), \mu_{b j}(t, s)=\mu_{b}(s)$. Formula (11) will be deduced by substituting the results above into (8).

$$
\begin{aligned}
& \eta(t, s)=\sum_{i=0}^{t} \sum_{j=0}^{s} p(i, j)\left[\frac{(i+S \alpha)}{(1+L \alpha)+\frac{i+S \alpha}{\mu_{o i}(t, s)+S \alpha}}+\frac{(j+S \alpha)}{(1+L \alpha)+\frac{j+S \alpha}{\mu_{o j}(t, s)+S \alpha}}\right] \\
& +\sum_{i=t+1}^{L-1} \sum_{j=s+1}^{L-1} p(i, j)\left[\frac{(i+S \alpha)}{(1+L \alpha)+\frac{i+S \alpha}{\mu_{b i}(t, s)+S \alpha}}+\frac{(j+S \alpha)}{(1+L \alpha)+\frac{j+S \alpha}{\mu_{b j}(t, s)+S \alpha}}\right] \\
& =\left[\sum_{i=0}^{t} V_{i} \frac{(i+S \alpha)}{\left.(1+L \alpha)+\frac{i+S \alpha}{\mu_{o}(t)+S \alpha}+\sum_{i=t+1}^{L-1} V_{i} \frac{(i+S \alpha)}{(1+L \alpha)+\frac{i+S \alpha}{\mu_{b}(t)+S \alpha}}\right]}\right. \\
& +\left[\sum_{j=0}^{s} H_{j} \frac{(j+S \alpha)}{(1+L \alpha)+\frac{j+S \alpha}{\mu_{o}(s)+S \alpha}}+\sum_{j=s+1}^{L-1} H_{j} \frac{(j+S \alpha)}{(1+L \alpha)+\frac{j+S \alpha}{\mu_{b}(s)+S \alpha}}\right] \\
& =\eta_{1}(t)+\eta_{2}(s)
\end{aligned}
$$

In this case, the optimal threshold vector $\left(t^{*}, s^{*}\right)$ should then meet:

$$
\left(t^{*}, s^{*}\right)=\operatorname{Arg} \max \left\{\eta_{1}(t)\right\}+\operatorname{Arg} \max _{t, s \in[0, L-1]}\left\{\eta_{2}(s)\right\}
$$


Formula (12) shows that, to get the threshold vector maximizing (9) is equivalen to get two thresholds maximizing two independent 1D reciprocal cross entropy functions which are decomposed by 2D reciprocal cross entropy function, respectively. Then, combine these two thresholds for the desired. Under typical circumstances, The computational complexity of solving the optimal threshold of $1 \mathrm{D}$ reciprocal cross entropy is $O(L)$. Therefore, the complexity of computing (12) is only: $O(L)+O(L)=2 O(L) \approx O(L)$.

\section{Case study and conclusions}

In order to verify the validity of the method, the surface defect images of the roller part which were shot by the CSP branch of a certain iron and steel enterprise are analyzed as a case. In this section, other three methods are also listed for comparison purposes. The results are shown in Fig. 4. The running time of 5 trials for each method are listed in Fig. 5. For comparison's sake, the optimal threshold for segmentation and the average running time are listed in Table 1.
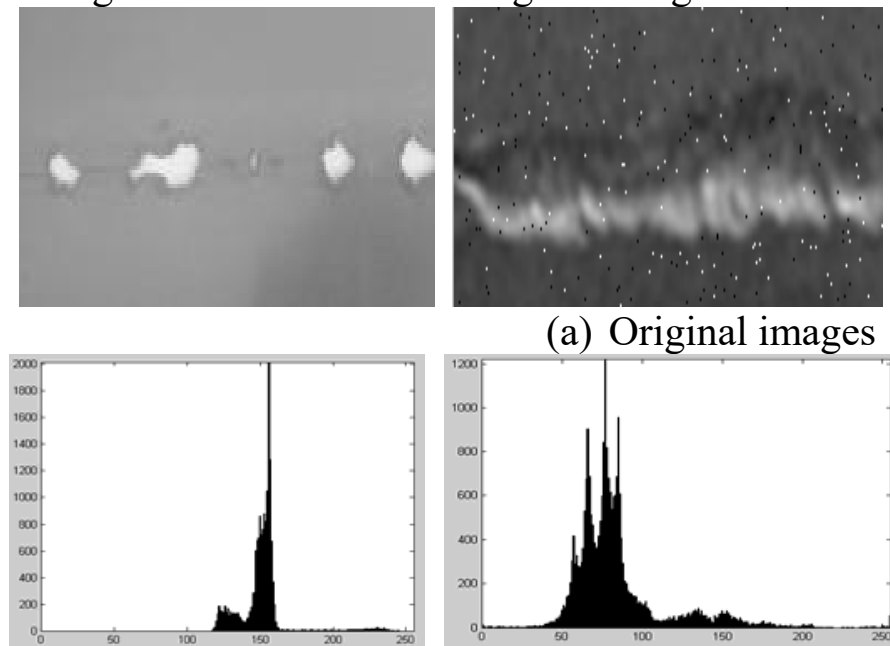

(a) Original images
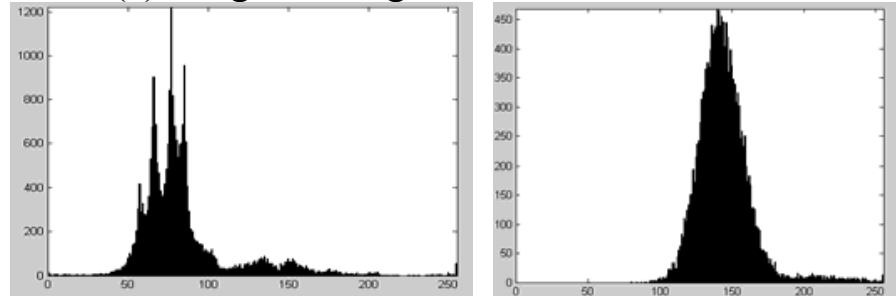

b) $1 \mathrm{D}$ histogram of the original images
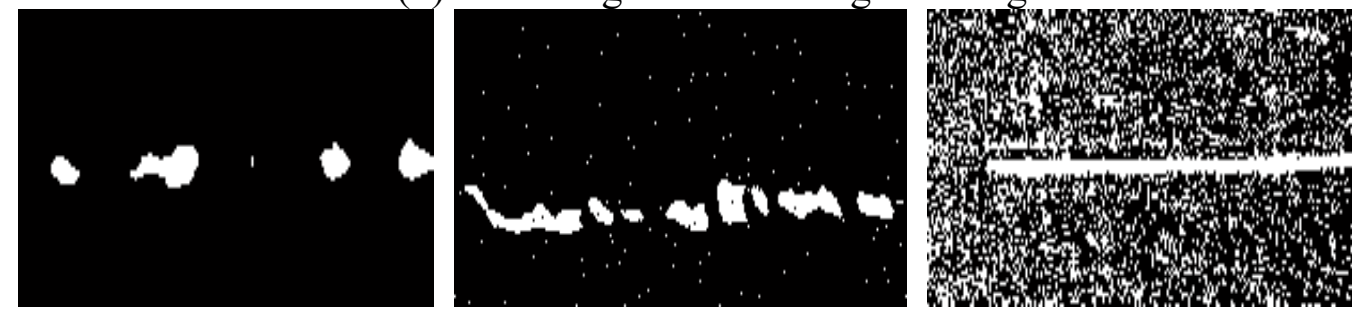

(c) The results of the method used in the literature [12]
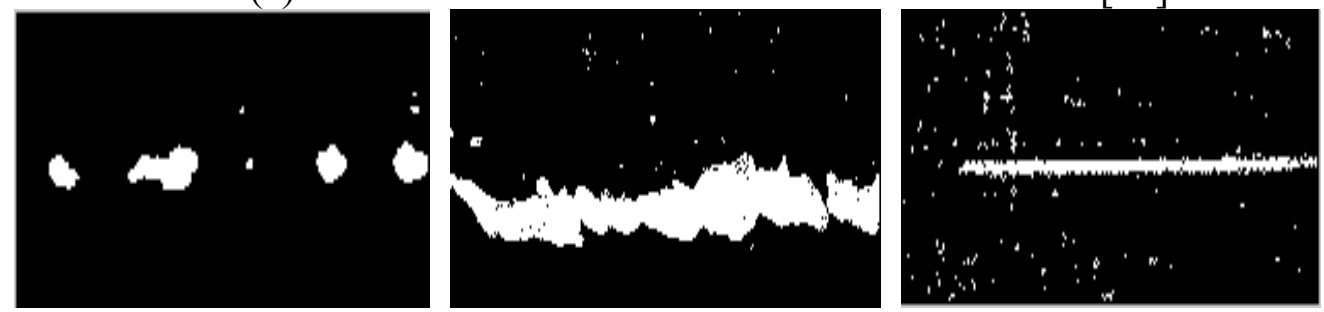

(d) The results of the method used in the literature [16]
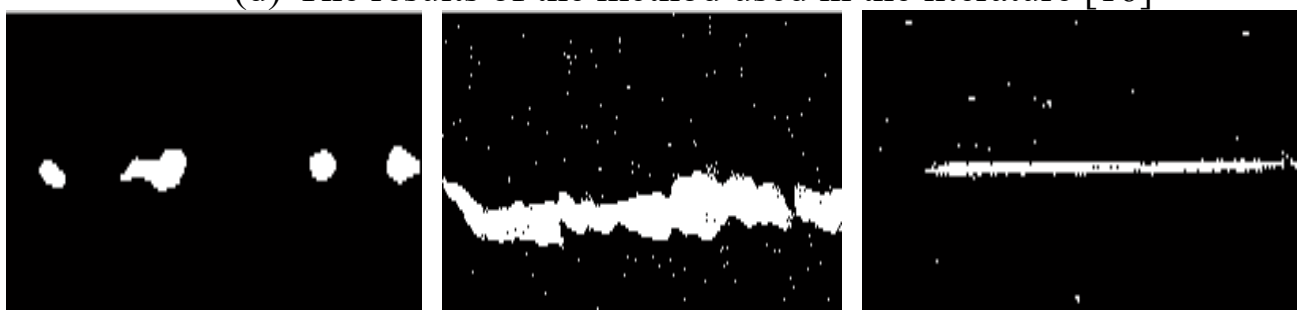

(e) The results of the method used in the literature [22] 

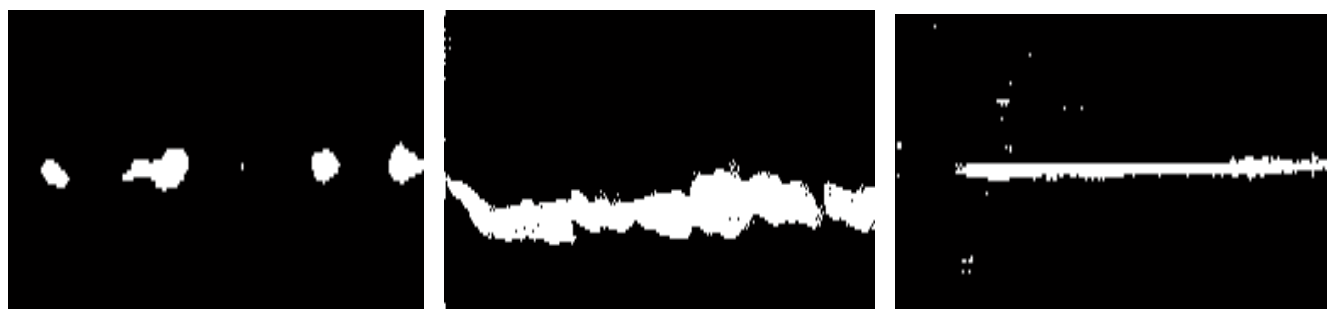

(f) The results of the method proposed in this paper

Fig. 4 Comparison of the results of each method

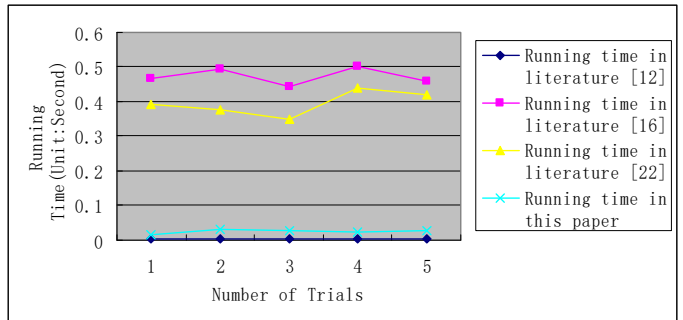

(a)
Running time for hole defect images in 5 trials images in 5 trials

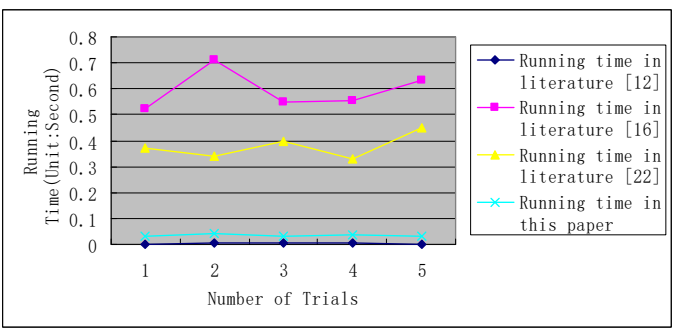

(b) Running time for surface spalling

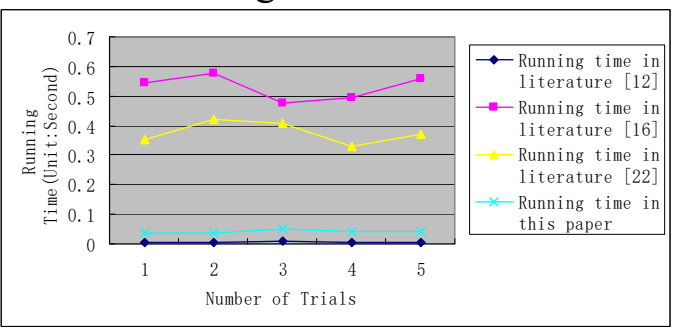

(c) Running time for surface scratch images in 5 trials

Fig. 5 Comparison of running time for each method

Table 1 Optimal threshold and average running time for each method Time unit: Second

\begin{tabular}{ccccccc}
\hline \multirow{2}{*}{ Method } & \multicolumn{2}{c}{ Hole defect images } & \multicolumn{2}{c}{ surface spalling images } & \multicolumn{2}{c}{ surface scratch images } \\
\cline { 2 - 7 } & threshold & ave. time & threshold & ave. time & threshold & ave. time \\
\hline Literature [12] & 174 & 0.00332 & 149 & 0.00282 & 141 & 0.00630 \\
Literature [16] & $(163,162)$ & 0.47310 & $(100,99)$ & 0.59430 & $(173,156)$ & 0.53120 \\
Literature [22] & $(230,182)$ & 0.39554 & $(112,151)$ & 0.37694 & $(154,183)$ & 0.37456 \\
This paper & $(182,142)$ & 0.02484 & $(108,106)$ & 0.03418 & $(148,182)$ & 0.04104 \\
\hline
\end{tabular}

Fig. 4(a) shows three original images used for the trials, the resolution of these images are uniformed. Among which, the left one is the hole defect image, the middle one is the surface spalling image, the right one is the surface scratch image. Aiming to test the anti-noise performance of the method, salt and pepper noise with a density of 0.01 is added into the surface spalling image, while gauss noise with a parameter of $(0,0.05)$ is added into the surface scratch image. Fig. 4 (b) shows one dimensional histogram of the three images. Fig. 4(c) (f) shows the results by adopting the method in literature [12], literature [16], literature [22] and this paper, respectively. It is noteworthy that the value of $\alpha$ in this paper meets the expression below.

$$
\alpha<0.01 \times \min \left\{\mu_{o}(t), \mu_{o}(s), \mu_{b}(t), \mu_{b}(s)\right\} / S
$$

As surface holes image is not mix with noise, and the illumination is relatively uniform, its 1D histogram shows a characteristic of double-peak. At this point, the effect of image segmentation of all methods are sufficiently close to each other. When mixed with salt and pepper noise in the image, its 1D histogram no longer has the characteristics of double-peak. The image in the middle of Fig. 4 (c) shows that the defect extracted from the original image is incomplete, and there are also a lot of noise in it. It is indicated that: (1) the salt and pepper noise interferes with the selection of threshold. (2) one-dimensional maximum entropy segmentation method is sensitive to salt and pepper noise. By using the method of literature [16], the defect extracted is relatively complete, but there are still some noise, shown as Fig. 4 (d). The main reason is that literature[16] uses neighborhood average gray value and the original gray value to establish the 2D histogram, this can suppress the salt and 
pepper noise to a certain extent. However, when the noise density is large, the anti-noise performance is significantly decreased. The same problem also exists in the method proposed by literature [22], shown as Fig. 4 (e). The defect extracted in Fig. 4 (f) is complete and the residual noise is greatly reduced. This demonstrates that the method proposed in this paper has a higher performance on the removal of salt and pepper noise than that of literature [16] and literature [22]. Similar conclusions can be made in the image with gauss noise, which is no longer introduced here. The method of this paper can be interpreted as: Firstly, finding a threshold to segment the object in the original image. Then, finding a threshold to filter the noise in the image filtered by utilizing morphological filter. Finally, the two thresholds are combined to get the desired. Since the method takes into account two kinds of gray information that before and after filtering, what's more, it retains more image spatial information. Therefore, compared with other methods, the anti-noise performance is improved obviously. Although the method in literature [12] is the fastest, the effect is the worst in the case of noise. The running time of the method in this paper is only about 5.5\% of that in literature [16]. This is mainly due to convert entropy calculation from logarithmic type to reciprocal type, and the dimension reduction method applied in searching the optimal threshold.

To sum up, the method in this paper is better than the other three methods in segmentation accuracy and noise immunity. In the premise of ensuring the complete segmentation of the object, the running speed is only $5 \% \sim 6 \%$ of the literature [16]. It can meet the needs of online real-time surface detection in a remanufacturing service system.

\section{Acknowledgment}

The first author wishes to acknowledge the financial support of the National Natural Science Fund Project of China: Research on Modulization and Adaptive Matching Mechanism of Reverse Supply Chain Services for Iron and Steel Industry (Project No. 71471143). Thanks for all the authors of the references who gives us inspirations and helps. The authors are grateful to the editors and anonymous reviewers for their valuables comments that improved the quality of this paper.

\section{References}

[1] E. Z. Li, P. J. Shi, B. S. Xu et al. Analysis of policies and regulations of China remanufacturing. Journal of mechanical engineering, 2015, 51(19):117-123.

[2] Mukherjee K, Mondal S. Analysis of issues relating to remanufacturing technology-a case of an Indian company Technology Analysis and Strategic Management, 2009, 21(5):639-642.

[3] X. Z. Hu, Y. Qiu. Nondestructive testing of pressure vessels: penetrant testing. NDT 2004, 26(7):359-363.

[4] X. W. Guo, R. S. Li, M. M. Ding. Simulating modulated thermography of cladding debond in solid rockets. Journal of mechanical engineering, 2011, 47(2): 9-15.

[5] Z. Y. Wang, Y. T. Liu, X. H. Wang et al. Application if infrared thermography in research of radar absorbing coating defects. Journal of aeronautical materials, 2012, 32(3):91-95.

[6] W. A. Tony. Automated Inspection of Metal Products Not Quite Ready for Prime Time. Iron and Steel Maker, 1992, 19(1):14-19.

[7] B. F. Yang, F. L. Luo, X. H. Cao et al. The detection of corrosion in aeroplane using pulsded eddy current nondestructive testing technology. Journal of test and measurment technology, 2005, 19(1):27-29.

[8] Jarvinen J. Real-time Surface Inspection of Steel Strips. Machine Vision News, 2002, 7:9-11.

[9] J. P. Yun, D. C. Choi, Y. J. Jeon et al. Defect inspection system for steel wire rods produced by hot rolling process. International Journal of Advanced Manufacturing Technology, 2014, 
70(9-12):1625-1634.

[10]H. Hu, Y. Li, M. Liu et al. Classification of defects in steel strip surface based on multiclass support vector machine. Multimedia Tools \& Applications, 2014,69(1):199-216.

[11]N. Otsu. A Threshold Selection Method from Gray-Level Histograms. IEEE Transactions on System, Man and Cybernetic, 1979, 9(1): 62-66.

[12]J. N. Kapur, P. K. Sahoo, A. K. C. Wong. A new method for gray-level picture thresholding using the entropy of the histogram. Computer Vision Graphics \& Image Processing, 1980, 29(3):273-285.

[13]A. S. Abutaleb. Automatic thresholding of gray-level pictures using two-dimensional entropy. Computer Vision Graphics \& Image Processing, 1989, 47(1):22-32.

[14]F. L. Chang, J. Liu, Y. Z. Qiao. Self-adaptive threshold segmentation for color image using two-dimensional entropy method based on genetic algorithm. Control and Decision, 2005, 20(6): 674-678.

[15]Y. T. Liu, M. Y. Fu. A Fast Infrared Image Segmentation Method Based on Two-Dimensional Entropy and Particle Swarm Optimization Algorithm. Pattern Recognition and Artificial Intelligence, 2008, 21(2):155-159.

[16] Y. J. Zhang, X. J. Wu, L. Z. Xia. A Fast Recurring Algorithm for Two-dimensional Entropic Thresholding for Image Segmentation. Pattern Recognition and Artificial Intelligence, 1997,10(3):259-264.

[17]X. M. Zhang, Y. B. Zheng. Precise 2-D Tsallis entropy image threshold segmentation and its fast recursive realization. Chinese Journal of Scientific Instrument, 2011,32(8):1796-1802.

[18]C. H. Li, C. K. Lee. Minimum cross entropy thresholding. Pattern Recognition, 1993, 26(4):617-625.

[19]J. H. Xue, Y. J. Zhang. Image Thresholding Based on Maximum Between-Class Posterior Cross Entropy. Journal of Image and Graphics, 1999, 4(2):110-114.

[20]Y. Q. Wu, Y. Song, H. C. Zhou. Boiler Coal Combustion Flame Image Multiple Thresholding Using Reciprocal Cross Entropy. Journal of Combustion Science and Technology, 2013, 19(6):493-500.

[21]Rafael C. Gonzalez, Richard E. (2011) Woods. Digital Image Processing(3rd Edition), Beijing: Publishing House of Electronics Industry, 2011.

[22]F. Yue, W. M. Zuo, K. Q. Wang. Decomposition Based Two-dimensional Threshold Algorithm for Gray Images. Acta Automatic Sinica, 2009, 35(7):1022-1027. 